\title{
Analysis of Subthreshold Transmission Characteristics for Gate Voltage and Doping Profiles of Asymmetric Double Gate MOSFET
}

\author{
Hakkee Jung \\ Department of Electronic Eng., Kunsan National University, \\ 558 Daehangno, Gunsan, Chonbuk, Rep. of Korea \\ hkjung@kunsan.ac.kr
}

\begin{abstract}
This study is to analyze the subthreshold transmission characteristics for the change of gate voltage of asymmetric double gate MOSFET. Asymmetric double gate MOSFET has the advantage of reducing short channel effects by controlling current flow by top/bottom gate voltage. Hermeneutical potential distributions were obtained by using Poisson's equation to analyze transmission characteristics by top/bottom gate voltage. Gaussian function was used as charge distribution. In order to analyze off-current and subthreshold swing, hermeneutic potential distributions were used. In conclusion, it was found that off-current depended on electron concentration affecting current flow, and subthreshold swing depended on the conduction path. Therefore, transmission characteristics of subthreshold were greatly affected by top/bottom gate voltage, which should be considered designing asymmetric double gate MOSFET.
\end{abstract}

Keywords: asymmetric double gate, subthreshold, conduction path, off-current

\section{Introduction}

Miniaturization of existing CMOSFET transistor has caused short channel effects such as movement of threshold voltage, increase of subthreshold swing, increase of offcurrent, and increase of drain induced barrier lowering, making it difficult to produce device with gate under $20 \mathrm{~nm}$ length. The transistor being developed to solve this problem is multigate MOSFET (Multi Gate MOSFET; MugFET) device [1]. The simplest structure among MugFETs is double gate (Double Gate; DG) MOSFET [2-3]. DGMOSFET can increase controlling ability of gate voltage for charges in channel by making gates on top/bottom layers, improving characteristics of subthreshold swing and movement of threshold voltage. Early DGMOSFET was mostly developed in symmetrical type, but the studies about asymmetric MOSFET which can design differently oxide thickness of top/bottom gate and applied voltage are being conducted actively [4-5]. In this study, with bottom gate voltage being parameters, off-current and subthreshold swing by top gate voltage were analyzed according to conduction path and electron concentration. Especially, unlike Ding et al. [5] using fixed value as charge distribution function in Poission's equation used in order to obtain hermeneutical potential distributions, the analysis closer to experiment was made by using Gaussian distribution function. Since variables of Gaussian distribution function, projected range and standard projected deviation are important factors deciding doping profiles, transmission characteristics of asymmetric DGMOSFET were analyzed investigating subthreshold swing and off-current for these two parameters. 


\section{Model for Off-current and Subthreshold Swing of Asymmetric DGMOSFET}

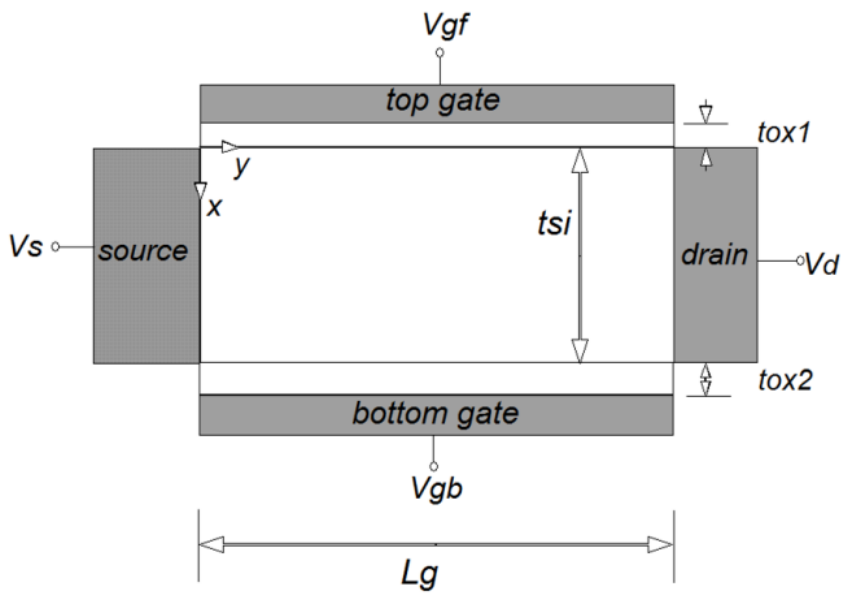

Figure 1. Schematic Sectional Diagram of Asymmetric Double Gate MOSFET

Figure 1 is schematic sectional diagram of asymmetric DGMOSFET. Unlike symmetrical DGMOSFET, it can apply top/bottom gate voltage differently by making gate structure of top and bottom layers differently. Also in this model, each oxide thickness of top and bottom layers can be designated differently, so parameters controlling short channel effects get increased.

First, when solving 2-dimensional Poisson's equation using boundary condition Ding et al. used, potential distributions of series form can be obtained as following.

$$
\phi(x, y)=V_{s}+V_{d} y / L_{g}+\sum_{n=1}^{\infty} A_{n}(x) \sin \left(n \pi y / L_{g}\right)
$$

Here $n$ is an integral number, $V_{s}$ source voltage, $V_{d}$ drain voltage, and $A_{n}$ was presented in bibliography [6].

One sixth of electrons in channel moving randomly with 3-dimensional free motion will head from source to drain, and the number of electrons reaching $t_{s i} W$ area of drain per unit time for channel width $W$ over potential barriers was, using Maxwell-Boltzmann statistics, $n_{m}=\left(n_{i}^{2} / N_{p}\right) e^{q \phi_{\min }\left(x_{e f f}\right) / k T}$. The $x_{\text {eff }}$ is conduction path and is the value of $x$ which can supposedly run most electrons, which can be obtained as follows.

$$
x_{\text {eff }}=\int_{0}^{t_{s i}} x \exp \left[\phi\left(x, y_{\min }\right) / V_{t}\right] d x / \int_{0}^{t_{s i}} \exp \left[\phi\left(x, y_{\min }\right) / V_{t}\right] d x
$$

The value of $y_{\min }$ is the value of $y$ when surface potential has the minimum, and $V_{t}=k T / q$. Off-current can be obtained as follows

$$
I_{\text {ther }}=\frac{q n_{m}\left(x_{e f f}, y_{\min }\right) t_{s i} W v_{t h}}{6}
$$


Here, $v_{t h}$ is thermal velocity.

The subthreshold swing can be defined as changed amount of top gate voltage when offcurrent decreases ten times. According to the definition, subthreshold swing for top gate voltage $V_{g f}$ can be presented as follows using Eq. (3).

$$
\begin{aligned}
& S=\frac{\partial V_{g f}}{\partial\left(\log _{10} I_{\text {ther }}\right)}=2.3\left[\frac{\partial \phi(x, y)}{\partial V_{g f}}\right]^{-1}
\end{aligned}
$$

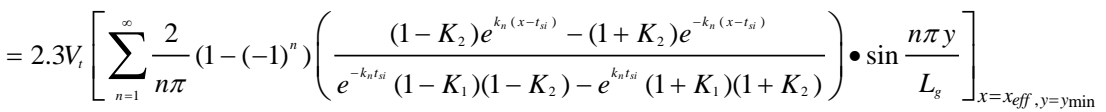

$$
\begin{aligned}
& k_{n}=n \pi / L_{g}, K_{1}=k_{n} \varepsilon_{s i} / C_{o x 1}, K_{2}=k_{n} \varepsilon_{s i} / C_{o x 2}
\end{aligned}
$$

Here, $C_{o x 1}$ and $C_{o x 2}$ are capacitance values of top/bottom gate oxides respectively. As shown in Eq. (3) and (4), the values of off-current and subthreshold swing changed by conduction path $x_{\text {eff }}$ value and the $y_{\text {min }}$ of minimum surface potential. This study is to analyze transmission characteristics of asymmetric double gate MOSFET by making an analysis of off-current and subthreshold swing according to top/bottom gate voltage and parameters of Gaussian function, projected range and standard projected deviation.

\section{Transmission Characteristics in Subthreshold Area of Asymmetric DGMOSFET}

Validity for the models of off-current and subthreshold swing presented in this study was already proven in previously published thesis [7]. So, in this study, transmission characteristics of subthreshold were observed by top/bottom gate voltage, projected range and standard projected deviation using models presented in chapter 2.

First, with projected range and bottom gate voltage being parameters, changes of transmission characteristics of subthreshold for top gate voltage were shown on Figure 2. As a result, it was shown that Figure 2(a) had a similar characteristic with Figure 2(c), Figure 2(b) having a similar characteristic with Figure 2(d). This means that subthreshold swing depends on conduction path and off-current depends on electron concentration in channel. Subthreshold swing was decreasing as top gate voltage got increased and bottom gate voltage got decreased. When it comes to conduction path, the more conduction path moved to top gate terminal, the smaller the value of subthreshold swing was. When bottom gate voltage increased, conduction path moved to bottom gate terminal, with the value of subthreshold swing increasing. When it comes to change of projected range, it showed a slight change in conduction path, but didn't affect subthreshold swing largely. The slope of off-current in Fig. 2(b) becomes a standard of subthreshold swing, and as the slope gets decreased, subthreshold swing will increase. Defining threshold voltage as top gate voltage when the off-current is $10^{-7} \mathrm{~A} / \mu \mathrm{m}$, threshold voltage decreased according to the increase of bottom gate voltage. As projected range increased, off-current decreased, by which threshold voltage increased, but the difference was very slight. 

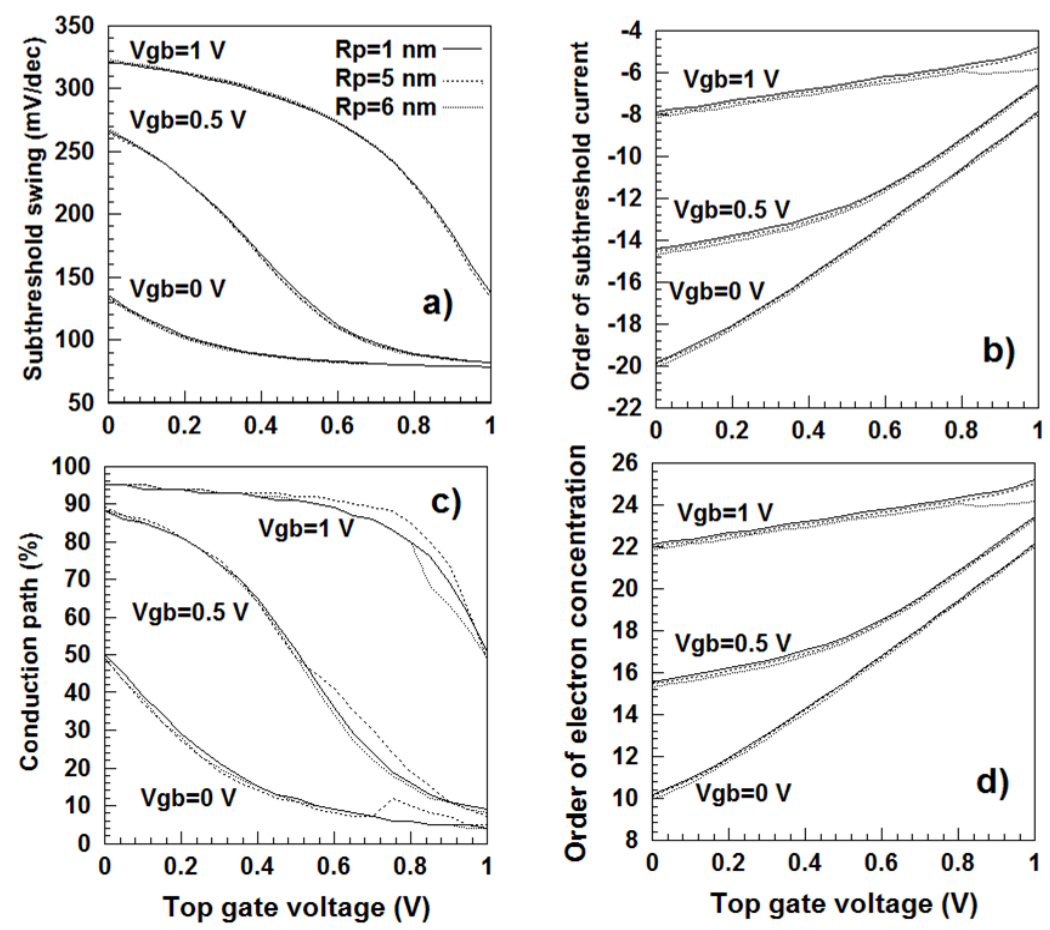

\section{Figure 2. Subthreshold Characteristics for Top Gate Voltage with Parameters of Projected Range and Bottom Gate Voltage a) Subthreshold Swing b) Order of Subthreshold Current c) Conduction Path and d) Order of Electron Concentration}

With standard projected deviation and bottom gate voltage being the parameters, the relation of transmission characteristics of subthreshold with the change of top gate voltage was shown in Figure 3. Compared with Figure 2, when not projected range but the standard projected deviation changed, transmission characteristics changed more. In case that top gate voltage was small in Figure 3(a) and 3(b), when standard projected deviation increased, conduction path moved to bottom gate terminal with subthreshold swing increasing. In case that top gate voltage increased, however, conduction path moved to top gate terminal when standard projected deviation increased, by which subthreshold swing also decreased. Besides, top gate voltage converting movement tendency of conduction path by standard projected deviation also changed according to bottom gate voltage. What's more, it was found that when bottom gate voltage got large, the change of the value of subthreshold swing got large. As known in Figure 3(b) for off-current, compared with Figure 2(b), the change of off-current by standard projected deviation was larger than the effect on the change of projected range. This effect was considered to be a result by electron concentration in Fig ure 3(d). Therefore, it was shown that standard projected deviation affected threshold voltage more largely. When bottom gate voltage increased, the reduction ratio of off-current in subthreshold was small, by which the value of subthreshold swing increased. 

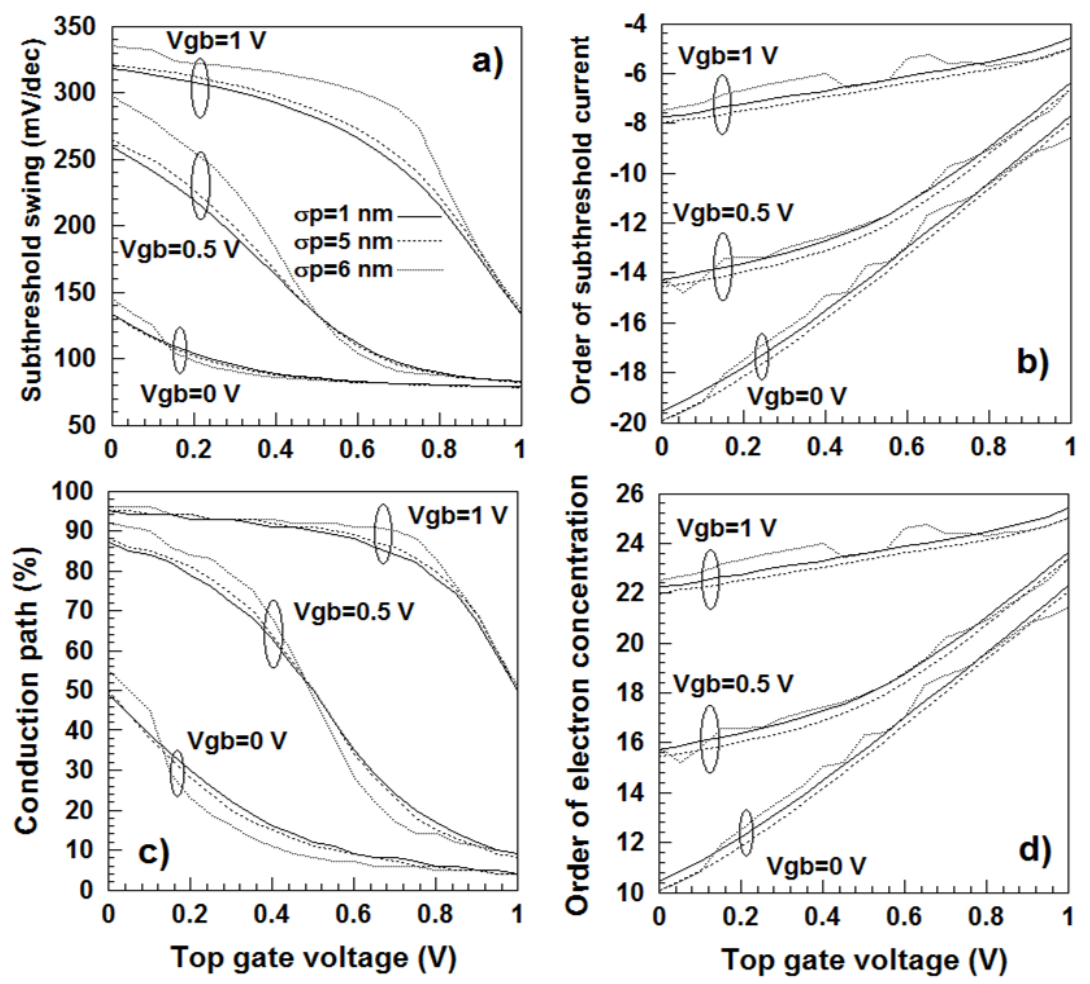

Figure 3. Subthreshold Characteristics for Top gate voltage with Parameters of Standard Projected Deviation and Bottom Gate Voltage a) Subthreshold Swing b) Order of Subthreshold Current c) Conduction Path and d) Order of Electron Concentration

\section{Conclusion}

This study analyzed the transmission characteristics of subthreshold for the change of gate voltage of asymmetric DGMOSFET. And characteristics in the range of subthreshold were observed according to top/bottom gate voltage, and projected range and standard projected deviation as parameters of Gaussian's function. As a result, offcurrent depended on electron concentration affecting current flow, and subthreshold swing depended on conduction path largely. As conduction path moved to top gate terminal, the value of subthreshold swing decreased. And as top gate voltage increased, conduction path headed for top gate terminal for bottom gate voltage to decrease. Like this, because the transmission characteristics were affected largely by bottom gate voltage and doping distribution function, it was thought that these factors should be considered to design asymmetric DGMOSFET.

\section{References}

[1] S. M. Lee and J. T. Park, "Electrical properties of nanoscale junctionless p-channel MugFET at cryogenic temperature”, J. Korea Inst. Inf. Commun. Eng., vol. 17, no. 8, (2013), pp. 1885-1890.

[2] J. B. Roldan, B. Gonzalez, B. Iniguez, A. M. Roldan, A. Lazaro and A. Cerdeira, "In-depth analysis and modelling of self-heating effects in nanometric DGMOSFETs", Solid-state electronics, vol. 79, (2013), pp.179-184.

[3] T. K. Chiang, "A new two-dimensional subthreshold behavior model for the short-channel asymmetric dualmaterial double-gate (ADMDG) MOSFETs", Microelectronics Reliability, vol. 49, (2009), pp. 693-698. 
[4] R. Vaddi, S. Dasgupta and R. P. Agarwal, "Analytical modeling of subthreshold current and subthreshold swing of an underlap DGMOSFET with tied independent gate and symmetric asymmetric options", Microelectronics J., vol.42, (2011), pp. 798-807.

[5] Z. Ding, G. Hu, J. Gu, R. Liu, L. Wang and T. Tang, "An analytical model for channel potential and subthreshold swing of the symmetric and asymmetric double-gate MOSFETs", Microelectronics J., vol. 42, (2011), pp. 515-519.

[6] H. K. Jung, "Analysis for Potential Distribution of Asymmetric Double Gate MOSFET Using Series Function”, J. Korea Inst. Inf. Commun. Eng., vol. 17, no. 11, (2013), pp. 2621-2626.

[7] H. K. Jung and O. S. Kwon, "Analysis of Channel Dimension Dependent Threshold Voltage for Asymmetric DGMOSFET", 2014 International Conference on Future Information \& Communication Engineering, vol. 6, (2014), pp. 299-302.

\section{Author}

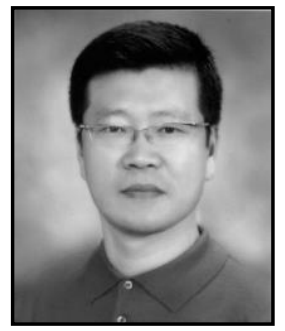

Hak Kee Jung, he received the B.S. degree from Ajou University, Korea, in 1983, the M.S. and Ph.D. degrees from Yonsei University, Seoul, Korea, in 1985, 1990, respectively, all in electronic engineering. In 1990, he joined Kunsan National University, Chonbuk, Korea, where he is currently a Professor in department of electronic engineering. From 1995 to 1995, he held a research position with the Electronic Engineering Department, Osaka University, Osaka, Japan. From 2004 to 2005, he was with the School of Microelectronic Engineering, Griffith University, Nathan, QLD, Australia. His research interests include semiconductor device physics and device modeling with a strong emphasis on quantum transport and Monte Carlo simulations. 